\title{
Criminologie
}

\section{La police : mythes et réalités}

\section{Jean-Paul Brodeur}

Volume 17, numéro 1, 1984

La police après 1984

URI : https://id.erudit.org/iderudit/017189ar

DOI : https://doi.org/10.7202/017189ar

Aller au sommaire du numéro

Éditeur(s)

Les Presses de l'Université de Montréal

ISSN

0316-0041 (imprimé)

1492-1367 (numérique)

Découvrir la revue

Citer cet article

Brodeur, J.-P. (1984). La police : mythes et réalités. Criminologie, 17(1), 9-41. https://doi.org/10.7202/017189ar d'utilisation que vous pouvez consulter en ligne.

https://apropos.erudit.org/fr/usagers/politique-dutilisation/ 
De même que la criminalité est la part de la déviance qui reçoit le plus d'attention de l'opinion publique et de ceux qui la façonnent, la police est l'agence de contrôle social dont l'action est la plus visible et la plus dramatisée. Cette première homologie n'est en réalité qu'un indice d'un phénomène beaucoup plus général et qui nous servira parfois de fil d'ariane dans notre enquête sur les appareils policiers. Comme l'ont souligné avec force des auteurs comme Black (1983) et Manning (1980), la criminalité et l'action déployée par l'État pour la contrôler peuvent être joints par des correspondances de structure si systématiques qu'elles paraissent souvent n'être que des images inversées l'une de l'autre. Ce dernier énoncé, dont nous fournirons plusieurs illustrations, doit s'entendre dans son sens descriptif et n'implique en lui-même aucun jugement de valeur sur les opérations de la police.

À titre d'illustrations initiales des correspondances auxquelles nous venons de nous référer, nous formulerons trois propositions relatives à la connaissance que l'on peut tenter d'acquérir de la police et de la criminalité.

A. Notre première proposition se formule comme suit: comme le comportement criminel, l'action policière est un objet qui oppose une résistance délibérée au projet de connaître. Cette résistance est d'une nature profondément différente des obstacles que rencontrent les efforts pour constituer un savoir dans les sciences de la nature et dans la plupart des sciences humaines: l'action policière, comme la délinquance, n'est pas un objet qui est disponible pour la théorie. Non seulement se dérobe-t-il ${ }^{1}$ mais, à la différence cette fois du comportement illégal, cette dérobade est parfois institutionnalisée par des législations comme la Loi sur les secrets officiels qui préservent la confidentialité des opérations.

* Professeur agrégé, Ecole de criminologie, Université de Montréal.

1. Les deux appendices d'Ericson (1981) sont éloquents sur les difficultes d'étudier la police. L'appendice $A$ contient une critique très vive des conclusions d'Ericson par le Bureau des commissaires (Board of Commissioners) qui l'avait initialement autorisé à effectuer sa recherche. Ericson réplique brièvement à ces critiques dans l'appendice B de son livre. Ericson est loin d'être le seul chercheur à avoir eu des démêlés avec un organisme policier: la publication des travaux de Greenwood et al. (1975) avait également suscité un tollé parmi les organisations policières et Greenwood a dô consentir à nuancer ultérieurement ses conclusions. Pour une discussion éclairée de ces problèmes, voir Van Maanen (1978). 
B. Notre seconde proposition désigne l'un des risques inhérents à l'étude des appareils policiers. À l'instar de Michel Foucault (1975), on a souvent dénoncé la fonction de diversion que remplit l'amplification des répercussions de la criminalité traditionnelle dans la presse, dans le discours politique et dans les idéologies qui légitiment et réclament une montée des châtiments ${ }^{2}$. Philippe Robert et ses collaborateurs $(1978,1980)$ ont réussi à montrer que les coûts tant économiques que sociaux de cette criminalité stéréotypée étaient sans commune mesure avec l'importance de ceux qu'engendrent des illégalismes moins bruyants comme la fraude fiscale ou la délinquance d'affaires. Des réserves analogues doivent être opposées à l'octroi précipité d'un privilège exclusif à l'étude de l'appareil policier public, qui n'est que le plus perceptible de nos systèmes de contrôle. On aurait tort de croire qu'il est le seul et surtout de postuler sans autre forme d'examen qu'il est le plus pénétrant. Par exemple, le contrôle exercé par la police sur les jeunes est négligeable par rapport à l'impact de l'encadrement que leur fournit l'appareil scolaire.

C. Notre dernière proposition préliminaire dicte la nature de la démarche que nous suivrons dans ce texte. Tant par rapport à la police que par rapport à la criminalité, il existe un écart considérable entre les résultats de la recherche et les évidences d'un sens commun qui est constamment dévoyé par les médias de masse. L'action policière, comme la criminalité, se donne à la perception à travers une mise en scène dramatique qui la travestit plus qu'elle ne la révèle. C'est pourquoi nous nous attacherons davantage à défaire certains préjugés, qui se sont montrés, à notre expérience, particulièrement persistants, qu'à retracer dans son détail l'évolution de la culture académique sur la police, dont les prémisses ne sont pas partagées par le public.

Nous n'en ferons pas moins état, dans une première section de cet article, des tendances les plus significatives de la recherche. Nous nous attacherons cependant plus longuement par la suite à réfuter un certain nombre d'affirmations répandues sur la police et dont la texture nous paraît ressortir davantage à une mythologie qu'à un savoir critique. Dans une dernière partie, la plus élaborée, nous nous efforcerons de substituer aux positions critiquées des orientations plus prometteuses.

2. Hall et al. (1978) constituent l'étude la plus élaborée qui ait été consacrée à ce phénomène de diversion. Elle porte sur la «panique morale» créée en Angleterre, dans les années soixante-dix, par l'amplification délibérée du phénomène de l'assaut dans les lieux publics (mugging). 


\section{LES TENDANCES RÉCENTES DE LA RECHERCHE}

Sans s'être livré à des calculs bibliographiques complexes, on peut affirmer avec une relative certitude qu'il n'y a pas de groupe professionnel qui ait fait l'objet d'autant d'études que les policiers. Une bibliographie exhaustive des écrits sur la police comprendrait plusieurs tomes de quelques centaines de pages. La partie la plus considérable de ces travaux a été effectuée dans les pays anglo-saxons et, de façon plus particulière aux États-Unis, où l'extrême décentralisation des corps policiers, qui excédaient le nombre de $40000^{3}$ a favorisé la poursuite d'études empiriques. Pour peu qu'ils fussent persévérants, les chercheurs ont toujours fini par trouver un corps policier prêt à les accueillir, alors que la pénétration au sein d'une grande bureaucratie d'État comme la Police nationale française a longtemps été soumise à des contraintes apparemment insurmontables ${ }^{4}$.

L'espace nous manque pour présenter une revue systématique de la masse des travaux publiés sur la police et nous renvoyons le lecteur à Feynaut et Van Outrive (1978), à Rumbaut et Bittner (1979) et à Sherman (1980); ces auteurs ont produit de solides analyses de la littérature récemment publiée. Pour ce qui est des instruments bibliographiques, mentionnons Shearing et al. (1979) pour le Canada, Whitehouse (1980) pour les États-Unis et Le Clère (1980) pour la France et l'Europe. On consultera également avec profit les Abstract on Police Science, publiés par la maison Kluwer (Pays-Bas) de 1973 à 1979; cette dernière publication a pris pour titre «Police Science Abstracts», à partir de 1980.

\section{A. DEUX CONSTANTES: L'ACCENT SUR LA VIOLENCE ET LE MAINTIEN DE L'ORDRE}

Il existe deux prises de position qui sont demeurées relativement inchangées, depuis les débuts de la recherche empirique sur la police, que l'on peut situer aux États-Unis, vers 1950, avec les tra-

3. Ce chiffre a été fourni par Reith en 1952 (voir la réimpression de son livre, Reith, 1975, p. 97). Il a été par la suite fréquemment confirmé, en particulier par la commission Katzenbach (United States, 1967). Les données les plus récentes (Skoler, 1977, p. 55) situent ce chiffre d quelque 20000 corps.

4. Depuis l'accession des socialistes au pouvoir, une vaste Étude auprès des personnels de la Police nationale a été réalisée en France par Hauser et Masingue (1982), deux chercheurs travaillant pour une firme privée (la société Interface). Hauser et Masingue (1983) explicitent les résultats de cette étude; celle-ci a donné lieu à la rédaction d'une Charte de la formation de la Police nationale, reproduite dans l'ouvrage précité. 
vaux de William Westley sur le corps de police de la ville de Gary, dans l'État de l'Indianas.

La première de ces positions tient dans l'établissement d'une relation constitutive entre le travail policier et les notions de violence et de coercition. Le caractère propre de cette relation entre la police et sa clientèle est sa symétrie ou, encore, sa réciprocité: les policiers étudiés par Westley (1970) et plus tard par Ker Muir (1977) et plusieurs autres perçoivent les situations au sein desquelles ils interviennent sous le sceau d'une menace qui leur est faite; de façon réciproque, toute une tradition sociologique américaine, dont l'un des représentants les plus actifs est Egon Bittner (1980a et b), voit dans le recours à la force coercitive le trait définitoire de la fonction policière $^{6}$. En France, Loubet del Bayle (1981), qui reprend la tradition weberienne, voit également dans la police l'appendice coercitif de l'État. Cette idée, est-il besoin de le mentionner, a reçu l'assentiment des criminologues radicaux qui se sont exprimés sur la fonction policière dans des recueils de textes colligés par Platt et Cooper (1974) et par le Center for Research on Criminal Justice (1976).

La seconde des constantes que nous avons relevées tient dans la négation soutenue que le rôle essentiel (ou principal) de la police, qu'on l'envisage en théorie ou en fait, soit de réprimer la criminalité. Le mandat de la police résiderait dans le maintien de l'ordre, cette dernière notion étant malheureusement entendue dans un sens assez diffus. L'un des premiers et des plus énergiques défenseurs de cette idée est James Q. Wilson (1968, 1982); elle est maintenant reprise avec vigueur par Peter Manning (1977; voir Manning and Van Maanen, 1978), l'un des chercheurs américains les plus influents, et par des théoriciens britanniques comme Evans (1974) et Alderson (1979). Les études empiriques conduites sur l'emploi effectif du temps de travail par la police apportent une confirmation réitérée à la thèse de Wilson?.

5. Bien qu'elle n'ait été publiée qu'en 1970 , l'étude de Westley a largement circulé après sa présentation en 1950, sous forme de thèse de doctorat. Westley (1953) contenait une présentation d'une partie des résultats de la thèse.

6. Bittner (1980b, p. 39) définit la police comme «un mécanisme pour la distribution de la force dans les situations sociales qui justifient une telle distribution". Bittner (1980a, p. 41) formule une définition similaire, en précisant que cette distribution de force coercitive ne fait pas l'objet d'une «négociation» entre la police et ceux qui font l'objet de cette distribution de force.

7. Pour les États-Unis, on peut citer les importantes études de Reiss (1971), de Greenwood et al. (1975) et de Black (1980). Les études les plus systématiques et les plus récentes sont, pour le Canada, celles d'Ericson $(1981,1982)$. Les rapports des com- 
On ne peut s'abstenir de remarquer l'existence d'une certaine tension entre les deux constantes dont nous venons de faire état. $L$ 'insistance sur la part de la violence et du danger dans la définition du contexte de l'intervention policière semblerait impliquer que ce contexte est de nature criminogène, sinon criminel. Or c'est très précisément cette implication que nie la seconde des constantes de la recherche, avec la conséquence que la légitimité du recours policier à la force est mise en question.

\section{B. LE DÉVELOPPEMENT DES ÉTUDES HISTORIQUES ET COMPARATIVES}

L'une des évolutions les plus prometteuses de la recherche sur la police réside dans la recrudescence de l'intérêt pour les études historiques et pour les analyses comparatives. Comme l'indique la réimpression récente d'ouvrages classiques comme ceux de Clément (1975) et de Chassaigne (1978), de même que la somme des sources textuelles et des travaux énumérés dans la bibliographie de Le Clère (1980), l'importance de pratiquer l'approche historique dans les études sur la police a depuis longtemps été reconnue, particulièrement en France. L'un des résultats féconds de l'impact du marxisme sur la criminologie aura été de réactiver l'intérêt pour les recherches historiques sur le contrôle social. Nous avons été témoins dans les années récentes de la publication d'ouvrages majeurs, comme les études de Williams (1979) et de Cameron (1981) sur la police française de l'Ancien Régime et celle de Leggett (1981) sur la Tchéka soviétique. En Angleterre, Bunyan (1976) a renoué avec une tradition d'ouvrages historiques sur la police qui remonte à Radzinowicz (1956) et Critchley (1967). Des études plus brèves comme la monographie de Pillorget (1978) sur Feydeau de Marville, les textes réunis dans Aubert et al. (1979), les travaux de Harring (1976) sur le développement de l'institution policière aux États-Unis et, enfin, ceux de Harring et McMullin (1975) sur l'histoire de la police de Buffalo (États-Unis) semblent en voie de se multiplier. Au Canada, les travaux de Lorne et Caroline Brown (1978), ainsi que ceux de MacLeod (1976), montrent qu'il est possible de traiter de l'histoire de la Gendarmerie royale du Canada (GRC) d'une manière autre qu'hagiographique. Jean Tur-

missions Ouimet (Canada, 1969, p. 42) et Hale (Ontario, 1972, p. 11-12) évaluaient déjà à plus de $80 \%$ le temps policier consacré à autre chose qu'au contrôle de la criminalité.

8. Feydeau de Marville fut Lieutenant général de la police de Paris entre 1740 et 1747. Â ce titre, il était chargé d'une part importante de la police de tout le royaume. 
mel $(1971,1974)$ a produit une esquisse de l'histoire de la police de Montréal, qui demeure utile.

L'extension du champ de la recherche dans le temps s'est également accompagnée de son élargissement dans l'espace. Nous nous limiterons à ne mentionner à cet égard que le nouvel influx qui a été apporté aux travaux comparatistes sur la police par la publication des recherches de David Bayley $(1969,1976)$ sur les services orientaux (police indienne et police japonaise) ${ }^{9}$.

\section{LA DÉTERRITORIALISATION DE L'OBJET-POLICE}

Le personnel d'un corps de police est en très grande partie composé par des policiers en uniforme, affectés pour la plupart à des tâches de patrouille. Il arrive même que de petits corps n'aient pas d'enquêteurs à leur service et qu'ils doivent faire appel à de plus gros corps voisins, lorsqu'il est nécessaire d'effectuer une enquête dans leur juridiction. Cette importance quantitative des patrouilleurs s'est reflétée dans les premières études empiriques sur la police, dont nous avons déjà dit qu'elles avaient porté, comme celle de Westley, sur de petits services de police. L'étude classique de A.J. Reiss (1971) a porté sur de grands corps policiers urbains aux États-Unis, mais son objet privilégié est toutefois demeuré le patrouilleur en uniforme. Cette circonscription de l'objet-police dans un groupe relativement homogène (les patrouilleurs) fut grosse de conséquences.

Elle engagea d'abord la croyance en la possibilité de produire une théorie intégrée de la police, l'homogénéité de l'objet retenu se réfléchissant dans l'unité de la théorie. Cette délimitation de l'objet détermina ensuite le privilège qui fut accordé aux tâches de maintien de l'ordre et de la paix dans l'identification de la nature de la fonction policière: le maintien de l'ordre est effectivement ce qui caractérise le poids des interventions du patrouilleur, à première vue. Elle donna enfin naissance à cette assez curieuse notion de sous-culture policière, qui n'est en réalité qu'un redoublement ethnologique naif du caractère uniforme des groupes étudiés (les patrouilleurs) ${ }^{10}$.

9. Bayley (1979) est une revue d'une partie des études historiques et comparatives qui ont été faites sur la police.

10. Les descriptions de ces sous-cultures varient considérablement selon les pays (comparables) et les méthodes employées. Ericson $(1981,1982)$, qui emploie des méthodes qualitatives telles que l'observation participante pour décrire des policiers canadiens, les représente, à l'instar de Westley $(1953,1970)$, cornme amers et intensément frustrés par la nature de leur travail. Reiner (1978), qui use du dépouillement de questionnaires pour étudier des policiers britanniques, dont la situation présente piusieurs analogies avec celle des policiers canadiens, nous apprend qu'ils sont motivés et satisfaits par leur travail. 
Or, à partir du milieu de la dernière décennie, on a assisté à une très importante déterritorialisation de ce qui constituait l'objet privilégié des recherches empiriques sur la police. Cet éclatement du champ a principalement consisté dans l'appréhension de nouveaux objets, notamment:

(i) les escouades d'enquêteurs, qui ont constitué la matière des importants travaux de Greenwood (1975) et de son équipe de la Rand Corporation, de ceux de Sanders (1977) et, pour le Canada, de ceux d'Éricson (1981);

(ii) les services politiques de sécurité de l'État, sur lesquels de vastes enquêtes gouvernementales - les commissions Church et Pike aux États-Unis et les commissions Keable, Kreever et McDonald au Canada, pour n'en énumérer que quelques-unes - ont jeté une lumière crue et dont les révélations ont donné lieu à une importante littérature;

(iii) les informateurs réguliers et les autres auxiliaires souterrains de la police, qui ont été le sujet d'un véritable travail de pionnier de la part de l'Américain Gary Marx (1974, 1980, 1981);

(iv) les agences de sécurité privée, dans l'étude desquelles une équipe de chercheurs regroupée autour de Clifford Shearing du Centre of Criminology de l'Université de Toronto s'est investie de façon particulièrement soutenue.

Nous examinerons plus loin si cette prise en charge de nouveaux objets ne contraindra pas les chercheurs à modifier l'allure générale de leurs théories sur les appareils policiers. On peut d'emblée répondre par l'affirmative à au moins un des aspects de cette question. Lorsque la patrouille formait l'objet privilégié des recherches sur la police, un certain consensus s'était formé pour concevoir cette dernière comme un service réactif, dont l'intervention était déterminée de l'extérieur. Ce consensus est maintenant remis en cause, l'étude de la mobilisation policière - ce qui déclenche l'intervention de la police - requérant maintenant de façon croissante l'attention des chercheurs.

\section{UN CHANGEMENT DE PERSPECTIVE: LA POLICE COMME ACCOMPLISSEMENT DE LA LÉGALITÉ}

Nous ne procéderons, à ce stade de l'exposé, qu'à une brève discussion d'un point, à notre avis fondamental, sur lequel nous comptons revenir dans la suite de cet article.

Nous commencerons donc par préciser que, dans le cours des années cinquante et soixante, plusieurs des travaux sur la police, 
effectués par des juristes comme Hall (1953, 1960), La Fave (1965) ou Davis $(1969,1975)$ se sont inscrits dans l'horizon d'une distinction explicitée par H.L. Packer (1964). Dans un article très influent, ce dernier a proposé de distinguer entre deux paradigmes de l'application de la loi (criminelle). Un premier paradigme, baptisé le "modèle du contrôle de la criminalité» (crime control model), était caractérisé par un primat accordé par le système de la justice pénale à l'efficacité de son fonctionnement aux dépens du respect de la procédure légale garantissant les droits individuels; le second paradigme, désigné comme le "modèle du respect de la procédure» (due process model), se définissait, comme son nom l'indique, par la primauté du droit des gens sur la fonctionnalité bureaucratique. Or la police apparut alors aux chercheurs comme le prototype d'une institution qui opérait selon le modèle du contrôle de la criminalité et qui usait de la discrétion dont jouissait ses membres pour rendre une justice expéditive et relativement indifférente aux droits légaux de sa clientèle. L'ouvrage classique de Jerome Skolnick (1966) Justice without Trial (Justice sans procès) est l'illustration la plus éloquente de cette perspective qui contrastait systématiquement le dérèglement des opérations policières avec les exigences méconnues de la lettre (et de l'esprit) de la loi. Des concepts tels que ceux de discrétion policière et de discrimination envers les minorités se voyaient octroyer un statut prépondérant dans ces recherches.

Or cette perspective a été récemment remise en question de toutes parts, comme en témoignent des travaux aussi différents que ceux de McBarnett (1979), Black (1980) et des criminologues qui s'inspirent du marxisme. La dichotomie établie initialement entre la légalité, prétendument favorable aux droits des personnes, et l'exercice discriminatoire du pouvoir policier est révoquée au profit de la reconnaissance capitale qu'au lieu de garantir les droits en limitant le pouvoir policier, la loi amplifie ce dernier en cautionnant son débordement. Loin donc d'affronter la lettre de la loi, le pouvoir de la police s'y adosse. Dans son livre récemment publié sur la police, Black $(1980$, p. xi, 2) définit le travail policier comme une instance de la loi elle-même.

\section{FRAGMENTS D'UNE MYTHOLOGIE}

Nous commencerons d'abord par préciser que la plupart des fragments que nous décrirons dans les prochaines pages ne composent pas exclusivement une mythologie policière que l'on pourrait qualifier de populaire. Certaines des positions dont nous ferons la critique sont parfois adoptées autant par des chercheurs que par des 
personnes qui ne possèdent pas d'expertise sur les questions policières. Cette remarque s'applique de façon particulière à la première des positions que nous interrogerons.

\section{A. UNE THÉORIE UNIDIMENSIONNELLE DE LA POLICE}

Nous reprendrons d'abord une idée dont nous avons précédemment dit qu'elle nous servirait de fil d'ariane, à savoir les homologies de structure entre la criminalité et le contrôle social, tel qu'il est exercé par la police. Walker (1977) met en cause de façon convaincante la possibilité de produire une théorie criminologique générale, qui fournirait l'explication de toutes les formes de déviance, celles-ci étant trop diversifiées pour recevoir le même type d'explication. Cet aperçu fondamental de Walker peut validement être rapporté à la sociologie de la police, dont nous doutons qu'elle puisse se produire sous la forme d'une théorie générale qui rendrait un compte unilatéral de toutes les manifestations de la réalité policière. Il importe en effet de distinguer entre au moins trois niveaux de réalité pour apercevoir l'amplitude de ces manifestations.

Un premier niveau, le plus abstrait, est constitué par la notion même ou le concept de police et leur champ de référence. Lorsqu'elle commença d'être plus fréquemment employée en France, vers le début du XVII' siècle, l'expression de police coïncidait avec celle du gouvernement ${ }^{11}$ et désignait toute façon contraignante de structurer l'espace social. La notion de police est aujourd'hui encore prégnante de sa généralité originelle et recouvre en puissance toutes les formes de l'autoritarisme ${ }^{12}$. La diversité et la complexité des manifestations de l'autoritarisme interdit qu'on les réduise à une essence commune.

Un second niveau de réalité, qui nous rapproche insensiblement du concret, est celui du prédicat - du qualificatif de policier; son emploi le plus exemplaire est de se conjoindre avec le substantif «État» pour former la redoutable expression d'Etat policier. Le qualificatif de policier semble alors moins général que la notion précédente de police, car il distingue parmi les mesures gouvernementales celles qui sont d'une nature répressive et qui ont explicitement pour

11. Le mot "police» vient du latin «politia» et du grec «politeia», qui signifient "gouvernement». Ces deux derniers mots dérivent de l'expression grecque «polis», qui veut dire «cité».

12. Donzelot (1977, p. 12), dans son ouvrage intitulé La police des familles, utilise cette notion dans son sens le plus large et se réfère d'ailleurs explicitement à une définition du concept de police proposée en 1768 par Von Justi (dans ses Éléments généraux de police). 
fin de perpétuer par la terreur ou l'intimidation la domination d'un groupe sur tous les autres. Il est toutefois capital de remarquer à cet égard que l'asservissement des membres du corps social est alors produit par l'action d'une pluralité d'appareils policiers, dont rien ne garantit qu'ils sont de même nature. Le dispositif policier mis en place dans l'Allemagne nazie, pour prendre un exemple connu, était composée de pièces relativement disparates, comme la Waffen SS, la Gestapo et la police judiciaire, qui continuait d'opérer comme sous la république de Weimar ${ }^{13}$. Or, il est très douteux qu'un appareil complètement militarisé comme la Waffen SS, une agence politique de sécurité d'État comme la Gestapo et un service traditionnel de police judiciaire possèdent suffisamment de traits en commun pour être uniment désignés, d'une autre façon que verbale, par le prédicat de policier.

Un troisième niveau de réalité est constitué par l'existence concrète des corps policiers. Or le phénomène le plus saisissant à ce niveau, si nous considérons le type de corps policier qui opère dans des sociétés occidentales comparables à la nôtre, est l'extrême diversité des tâches qu'ils sont conduits à assumer, cette variété d'attributions étant par surcroît modalisée par leur théâtre d'opération (un grand corps policier urbain est une organisation qui diffère profondément d'un petit corps opérant dans un milieu rural, en assumant, entre autres, les fonctions d'un service d'incendie). Cette diversité n'est pas telle qu'on ne puisse au moins tenter de formuler quelques énoncés généraux sur les caractéristiques des corps policiers. Elle interdit toutefois qu'une sociologie de la police adopte une attitude réductrice, espérant décomposer la complexité de son objet en quelques dimensions simplificatrices. Comme le remarque avec justesse Marenin (1982), on ne voit pas comment l'application des règlements de la circulation s'intègre au cadre présumé d'une stratégie de mise en tutelle du prolétariat.

Nous sommes parvenu à une même conclusion sur la nécessité de concevoir la sociologie de la police comme une entreprise ouverte et pluridimensionnelle au terme de notre examen de chacun des niveaux de réalité que nous avons distingués. Cette conclusion s'im-

13. Pour le fonctionnement de la justice sous le troisième Reich voir Grunberger (1974, p. 155-168). Pour l'organisation des services répressifs sous Hitler, voir la magistrale étude de Wormser-Migot (1968: les deux premiers chapitres). Selon cette étude, les services de police criminelle dépendaient du ministère de la Justice et ceux de la police politique du ministère de l'Intérieur (pp. 46-47). D'après Wormser-Migot (p. 231), «on peut dire que de mars 1942 a avril 1945 environ 45000 Waffen S.S. ont servi dans les camps» de concentration. 
pose de façon d'autant plus impérieuse que la réalité policière intégrale est constituée par la somme de ces trois niveaux.

\section{B. UN MONOPOLE PROBLÉMATIQUE: CELUI DE LA VIOLENCE}

Il est une affirmation sur la police que l'on entend si souvent qu'elle a maintenant pris la figure d'un truisme qui n'est jamais interrogé. Nous en avons fait état dans notre brève revue de littérature. La police serait censée disposer du monopole légal du recours à la violence physique. Ce monopole distinguerait la police des autres appareils et constituerait le cour profond de cette institution. Cette concession d'un monopole est, au vrai, hâtive et elle exprime une position qui nous semble de plusieurs façons incorrecte.

Il existe d'abord au sein du système de la justice pénale d'autres fonctionnaires qui sont légalement habilités à user de coercition physique contre autrui: les gardiens de prison. On sait en outre qu'un nombre croissant d'employés d'agences de sécurité privée et de constables spéciaux de toutes sortes sont autorisés à porter et, dans certaines conditions, à utiliser des armes. Certaines agences de sécurité privée sont spécialisées dans l'utilisation de chiens, bêtes absolument féroces, pour intimider des groupes de manifestants et, en particulier, des groupes de grévistes.

Ces remarques sont tellement obvies qu'on s'étonne de devoir les faire. On pourrait en outre nous rétorquer que les groupes mentionnés appartiennent à des services para-policiers dont le lien avec le monde de la justice pénale est immédiat. Le monopole légal de l'exercice de la violence demeurerait l'apanage du système de la justice pénale, sinon de la police. Or cette thèse est fausse, serait-elle même élargie de façon weberienne à ne valoir que de l'État, considéré en lui-même.

Comme l'a remarqué Bittner (1980b, p. 8), il est en effet une profession qui partage d'une façon hautement significative ce présumé monopole légal de l'exercice de la violence physique: c'est la profession médicale, dans une multitude de ses attributions (la pratique de la chirurgie, le recours à certains modes de thérapie comme l'utilisation psychiatrique d'électrochocs, l'emploi de drogues et d'autres produits chimiques dont les effets sur l'organisme sont violents, etc.). Notons à cet égard qu'infliger des lésions corporelles à des fins médicales est explicitement autorisé par l'article 45 du Code criminel canadien. Il y avait jusqu'à récemment au Canada un autre groupe de personnes, important en son nombre, qui jouissait d'une immunité légale par rapport à l'exercice d'une forme non bénigne de 
violence: il s'agissait des maris qui n'étaient pas légalement imputables du viol de leur femme (cette situation a été modifiée par la récente refonte des dispositions légales sur les infractions de nature sexuelle).

Il ne fait pour nous aucun doute qu'un examen plus attentif pourrait révéler encore mieux à quel point le monopole légal de la violence qu'on attribue à la police n'est qu'une fiction réconfortante. L'article 43 du Code criminel, par exemple, confère aux parents, à ceux qui les remplacent et aux instituteurs le droit d'employer la force («raisonnable») pour corriger selon le cas un enfant ou un élève. Un droit similaire est octroyé par l'article 44 du même code à un capitaine de navire, pour faire régner la discipline sur son bateau. L'article 81 du Code criminel autorise explicitement les combats de boxe, dont on sait qu'ils conduisent parfois à la mort de l'un des adversaires. Cette liste d'exemples pourrait être encore allongée; nous nous en tiendrons à ces remarques qui sont largement suffisantes pour notre critique de la thèse du monopole policier de la violence.

\section{UNE CLAIRVOYANCE ILLUSOIRE}

Le troisième des fragments mythologiques que nous désirons présenter est sans doute celui qui mérite le mieux cette appellation, car il est largement le produit de la littérature policière dans sa variante traditionnelle - le roman policier et ses dérivés audiovisuels - ou sa variante plus récente - le roman d'espionnage et ses sous-produits. Les effets mystifiants de cette littérature sont nombreux.

La littérature policière procède d'abord à une inversion complète de la réalité policière: à très peu d'exceptions près - comme les romans de Joseph Wambaugh, lui-même un ancien policier -, cette littérature est tout entière consacrée à l'enquête policière, qui ne constitue qu'une partie très restreinte de l'activité de la police. La littérature d'espionnage, quant à elle, déculpe les capacités de surveillance de l'appareil policier: rien n'échapperait à son regard, à moins qu'il ne choisisse pour des motifs occultes de se couvrir.

Dans sa célébration de l'enquête, la littérature policière métamorphose une procédure marquée par l'errance en l'itinéraire d'une découverte, au terme duquel la force déductive de l'enquêteur, s'appuyant sur des indices matériels sublimés par la magie des laboratoires de police technique, parvient à débusquer un infracteur, dont l'identité était jusque-là inconnue. Or il n'est, au regard des recher- 
ches empiriques faites sur l'enquête policière, aucune représentation qui soit plus éloignée du travail effectif de la police que celle qui est diffusée dans l'opinion publique par la littérature policière. Les affaires sont systématiquement réglées par une procédure dont la nature est contraire à celle qui est fabriquée par l'imagerie littéraire: rarement découverte par le raisonnement de l'enquêteur ou l'expertise des laboratoires, l'identification du coupable est habituellement le produit d'une dénonciation faite à la police. Cette dénonciation provient soit i) de la victime elle-même, ii) d'un ou de plusieurs témoins, iii) d'un informateur au service de la police et iv) de l'infracteur lui-même, qui révèle son crime à la police ou qui, dans des cas relevant moins d'infractions à la loi que de non-conformisme politique, attire sur lui l'attention des services de sécurité en affichant bruyamment sa dissidence ${ }^{14}$. Le reste des affaires est surtout réglé par l'arrestation du prévenu en flagrant délit ou dans le cours d'une fuite avortée et, enfin, au hasard d'un ratissage, souvent relié à une infraction aux règlements de la circulation. Ces dernières opérations sont la plupart du temps effectuées par les patrouilleurs.

Ces remarques n'ont pas pour but de déprécier le travail de la police et nous reconnaissons qu'il existe des enquêtes qui sont brillamment conduites. On trouvera l'exemple d'une telle enquête dans Québec (1980, pp. 196-201). Il nous apparaît important d'adopter une vue sobre des capacités de la police pour ne pas entretenir des attentes irréalistes à son endroit: comme en témoignent des affaires comme l'égorgeur du Yorkshire, celles du tueur d'Atlanta ou de l'assassinat récent dans l'Ouest du Canada de toute une famille de villégiateurs, une police à qui on ne désigne pas explicitement ses coupables est livrée à l'interminable errance de l'enquête à laquelle seul le hasard met un terme.

\section{POLICE OU POLITIQUE: UNE ALTERNATIVE TROP SIMPLE}

Il est deux thèses sur la nature des relations entre l'appareil policier et le pouvoir de l'État qui s'expriment dans des formules en apparence opposées. Selon la première de ces formules, la police constituerait un «État dans l'État»; selon la seconde, elle formerait le «bras armé de l'État». Le premier de ces slogans énonce ce que nous appellerons la thèse de l'insularité, qui sert souvent d'alibi au pouvoir politique pour excuser les insuffisances du contrôle qu'il exerce sur les corps policiers: l'appareil policier est conçu comme une

14. Pour des données récentes sur les façons d'identifier les suspects, voir Ericson $(1981$, p. 136). 
instance autonome qui résiste victorieusement aux contraintes extérieures pour poursuivre son propre intérêt. Le second de nos slogans exprime la thèse dite instrumentale: la police serait un instrument relativement inerte qui s'animerait pour répondre mécaniquement aux commandes de l'État, lui-même au service des intérêts de la classe dont il est le mandataire. Cette thèse s'énonce sous diverses variantes dans les travaux des criminologues influencés par le marxisme, comme par exemple Harring (1976), Hepburn (1977) et d'autres travaux déjà cités.

Il faut, par rapport à ces deux thèses, reconnaître sans ambages qu'elles peuvent être illustrées par l'histoire des corps policiers. Ainsi, il semble incontestable que tout le domaine du contrôle des informateurs est une chasse policière gardée qui résiste farouchement à toute intrusion extérieure. De façon converse, on ne saurait crédiblement nier que la police provinciale québécoise s'est comportée comme la brute de Maurice Duplessis pendant la célèbre grève de l'amiante de 1949. La polyvalence de la réalité policière assure en effet d'emblée qu'on puisse y trouver matière à illustrer toutes les positions. On se tromperait toutefois lourdement en confondant l'illustration d'un propos avec la démonstration d'une thèse. En tant qu'affirmations générales qu'on ambitionnerait de rapporter à la totalité de la réalité policière, les thèses de l'insularité et de l'instrumentalité des corps policiers sont précisément le produit de cette confusion simplificatrice entre les procédures d'illustration et de démonstration. L'une ou l'autre de ces thèses sont incapables de rendre intégralement compte de leur objet, que ce soit au niveau des faits empiriques ou à celui de la rectitude des concepts.

L'espace nous manque pour énumérer tous les faits empiriques qui contredisent les deux thèses que nous discutons. Nous ne mentionnerons donc que les principaux butoirs, limitant nos exemples au contexte canadien. La thèse de l'insularité se heurte à l'intervention systématique du politique dans l'orientation et souvent également dans l'administration des activités de la police. Cette intervention se produit d'abord d'une façon régulière par la promulgation des diverses lois et réglementations qui affectent l'action de la police. Elle se produit en outre de façon irrégulière, mais de plus en plus fréquente, par l'institution de mesures de contrôle d'exception comme les commissions d'enquête. Comme l'a montré Tardif (1974), enfin, elle s'exerce de façon diffuse par des pressions de toute nature sur la hiérarchie policière. Au regard de l'immixtion du politique, la police constitue bien davantage un cirque qu'une île. 
C'est, paradoxalement, en partie les mêmes faits qui peuvent être allégués pour falsifier la thèse instrumentale. En même temps qu'elles constituent une intervention dans les affaires de la police, les commissions d'enquête ont inlassablement fait la preuve de la laxité des contrôles qu'exerçaient les autorités légitimes sur les activités policières. Il est toutefois un autre obstacle factuel sur lequel vient se briser de façon irrévocable la thèse de l'instrumentalité: c'est celui du syndicalisme policier. Il faut volontairement consentir à s'aveugler pour persister à penser, en dépit de grèves répétées et couteuses, que les policiers constituent un groupe de tâcherons passifs, qui sont docilement soumis aux volontés des élites politiques.

On peut toutefois invoquer un argument plus général contre ces thèses de l'insularité et de l'instrumentalité. Elles ne constituent au vrai que deux radicalisations inversées d'une position qui repose sur un présupposé unique. Il faut en effet que l'État et l'appareil policier soient respectivement conçus comme des termes jouissant d'une forte cohésion pour entretenir des rapports aussi élémentaires que l'autarcie ou la subordination. Il est, pour prendre un exemple simple, difficile de concevoir les relations entre un État décentralisé et la myriade de ses corps policiers à travers les notions globalisantes de l'insularité ou de l'instrumentalité: chacun des corps policiers considérés entretiendra une relation qui lui est propre avec l'autorité politique dont il relève, la résultante de cet enchevêtrement de rapports différents étant plus près d'un chaos bureaucratique que d'une moyenne numérique.

De trois choses, en effet, l'une. (i) Ou bien le présupposé de cohésion ne sera pas respecté - comme par exemple, en Amérique du Nord, où tant le gouvernement que la police sont fractionnés en une multitude de juridictions souvent en rivalité - , auquel cas prévaudra une situation semblable à celle que nous avons décrite à la fin du paragraphe précédent. (ii) Ou bien le présupposé de cohésion interne des appareils est satisfait - notons que cette hypothèse comporte une large part de spéculation ${ }^{15}$-, auquel cas il s'établit entre

15. Un pays comme la France est, par exemple, caractérisé par un très haut niveau de centralisation policière: il n'y existe que deux grandes organisations policières: la Police nationale et la Gendarmerie, auxquelles s'ajoutent quelques services de renseignement et de contre-espionnage, dont les désignations varient fréquemment. Or cette étroite centralisation n'empêche pas que les diverses pièces de ce dispositif policier ne reproduisent un état de conflit à l'intérieur de la structure qui les réunit (la "guerre des polices» a fait l'objet d'une littérature assez considérable, en France). Même dans les meilleures conditions formelles, l'hypothèse de la cohésion des services demeure problématique. 
l'État et sa police un équilibre pragmatique qui se démarque antant de la fronde que de l'asservissement. (iii) Ou bien, enfin, cet équilibre est rompu, auquel cas l'un des termes tend à s'assimiler entièrement l'autre, l'idée même d'une relation étant abolie: l'État devient une police totale ou la police dégénère en une simple milice gouvernementale. Dans tous les cas, les notions d'insularité et d'instrumentalité sont inadéquates pour penser les rapports entre la police et l'autorité politique. Ajoutons qu'à l'intérieur de la criminologie radicale, Humphries et Greenberg (1981), Spitzer (1981) et Beirne (1979) ont soumis la perspective instrumentale à une critique acérée.

\section{E. LES MALENTENDUS DE LA LÉGALITÉ}

Peu de matières auront fait l'objet d'autant de revendications bien pensantes que l'exigence de soumettre la police à l'observance des lois. Comme nous l'avons déjà laissé entendre dans la trop courte revue de la littérature que nous avons entreprise, cette question a constitué le centre d'un débat majeur, auquel nous nous proposons de contribuer. Nous commencerons à cet égard par baliser le terrain en indiquant que cette notion de légalité policière abrite une complexité insoupçonnée.

Le leitmotiv d'une certaine pensée éditorialiste est que la police ne devrait pas être «au-dessus des lois». Par quoi l'on veut dire que la loi devrait contraindre également tous les citoyens, l'égalité se manifestant dans l'application méthodique d'une sanction à tout infracteur aux lois, quel que soit son statut. Ces remarques simples, qui prétendent en outre exprimer un idéal démocratique, se fondent sur un ensemble de postulats, dont la vérité est tenue pour acquise. Soit:

(i) Le postulat de cohérence: la loi est suffisamment cohérente pour qu'il soit possible d'en observer en même temps toutes les dispositions (satisfaire à un article n'implique jamais déroger du même coup à un autre).

(ii) Le postulat d'uniformité: toutes les lois pénales sont de même nature: elles énoncent des prohibitions, auxquelles sont soumis sans distinction tous les membres d'une société; les lois sont en outre de même niveau, l'idée d'une hiérarchie juridique selon laquelle des lois de niveau supérieur modalisent l'observance de certaines lois de niveau inférieur n'étant pas envisagée.

(iii) Le postulat de la décision: il est toujours possible de trancher sans ambiguilté si une loi a été respectée ou violée. 
(iv) Le postulat de l'univocité sémantique: le langage juridique est caractérisé par l'identité de sens des expressions qu'il utilise, quel que soit le contexte de leur emploi. Ainsi, lorsqu'on se demande si $\mathbf{A}$, un agent de police infiltré, et $\mathrm{B}$, qui est le bras droit du chef d'une bande de kidnappeurs, sont coupables de complicité dans l'élaboration d'un projet d'enlèvement, on poserait par rapport à ces deux personnes la même question, caractérisée par la constance du contenu sémantique de l'expression "complice».

Dans le cas du dernier postulat énoncé, nous croyons qu'il suffit de produire, comme nous l'avons fait, un exemple problématique pour indiquer que le postulat est faux. Il n'est en réalité aucun de ces postulats qui ne soit incorrect, comme nous tenterons de le montrer lorsque nous reprendrons la question de la légalité policière dans la prochaine section de cet article.

\section{DIRECTIONS ALTERNATIVES}

Nous allons soumettre dans cette partie finale de notre article une série de propositions qui font pendant aux positions que nous avons critiquées dans la partie précédente.

\section{A. L'ABANDON DU VOLONTARISME ÉPISTÉMOLOGIQUE}

Les propositions que nous formulerons seront marquées par deux traits. D'abord par leur essentielle ouverture: quoiqu'elles prendront la figure d'énoncés généraux, elles ne constituent que des indications sur des recherches à accomplir sur un objet dont nous avons reconnu le caractère bigarré.

Il est une autre propriété de cet objet, dont nous nous proposons de tenir un compte explicite: la police n'est pas un ensemble de choses qui s'exhibent mais un groupe de personnes qui agissent. Or, il est capital pour une sociologie de l'action de reconnaître que dans la mesure où la pratique humaine est ordonnée par des fins, elle s'incarne sous la forme d'une stratégie pour laquelle l'opacité et l'ambiguïté constituent une dimension délibérément recherchée et entretenue; cette dimension résiste de manière irréductible à une objectivation théorique complète (la transparence est la négation même de la stratégie, qu'elle assimile à un plat comportement). C'est donc une erreur profonde que de s'obstiner à épingler sur un schéma rationalisant une activité qui conçoit son incertitude comme l'un de ses atouts décisifs. Cette erreur engendre de brutales déconvenues lorsqu'elle 
se double de la volonté de raidir davantage ces schémas en leur conférant la pesante traduction d'une législation.

\section{B. LA FORCE DE L'IMAGINÉ}

Dans l'histoire de la police, il est quelques figures marquantes comme Sir Robert Peel, pour l'Angleterre, et les lieutenants généraux de police d'Argenson, Sartine et Lenoir, pour la France. Peu de personnages ont toutefois joui de la réputation d'exercer un pouvoir aussi légendaire que celui de Joseph Fouché, le ministre de la police de Napoléon Bonaparte. Il suffit de lire les pages que Sir Leon Radzinowicz (1956) consacre à l'histoire de la police française pour mesurer à quel point une aura de puissance occulte s'attache au nom de Fouché, dont les agents étaient censés s'être infiltrés dans tous les secteurs de la vie française. Or Fouché lui-même, dans ses Mémoires parus en 1824, s'est expliqué sur la façon dont il a réussi à défendre le pouvoir de Napoléon contre la subversion interne à l'Empire:

... j'administrai bien plus par l'empire des représentations et de l'appréhension que par la compression et l'emploi des moyens coercitifs; j'avais fait revivre l'ancienne maxime de la police, savoir: que trois hommes ne pouvaient se réunir et parler indiscrètement des affaires publiques sans que le lendemain le ministère de la Police n'en fût informé. Il est certain que j'eus l'adresse de répandre et de faire croire que partout où quatre personnes se réunissaient, il se trouvait, à ma solde, des yeux pour voir et des oreilles pour entendre. Sans doute une telle croyance tenait aussi à la corruption et à l'avilissement général...

Ainsi la voilà connue, cette grande et effrayante machine appelée police générale de l'Empire... (Fouché, 1945, p. 227-228, nous soulignons).

Des études historiques sur Fouché - Madelin (1930), Tulard (1979) et Arnold (1979) - ont confirmé que son pouvoir reposait plus sur son habileté à jouer des craintes de ses contemporains que sur les effectifs et les ressources matérielles dont il disposait. L'étude de Williams (1979, p. 95) sur la police de l'Ancien régime est parvenue aux mêmes conclusions: l'effroi que suscitaient d'Argenson surnommé «le Damné» par ses contemporains - et Sartine - il est l'inventeur de l'ancienne maxime sur la police que Fouché se vante d'avoir fait revivre - était sans proportion avec l'importance matérielle de l'appareil policier qu'ils dirigeaient.

Ces remarques ont une pertinence immédiate pour comprendre la police de notre époque. Les révélations des commission d'en- 
quête sur les méthodes utilisées par le FBI ${ }^{16}$ et la $\mathrm{GRC}^{17}$ pour réprimer la dissidence politique et lutter contre le terrorisme montrent à quel point la manipulation des appréhensions et des rumeurs et, pour parler dans le jargon des services secrets, le recours à la désinformation (en anglais disinformation: diffusion systématique d'une fausse information) constituent des tactiques dont l'emploi est beaucoup plus régulier que le recours à la force physique. D'une façon analogue, les études déjà citées sur la patrouille policière, en particulier celle de Ker Muir, ont souligné que le problème que devait quotidiennement résoudre le patrouilleur dans le maintien de l'ordre était de conserver une image suffisamment intimidante pour inhiber chez le citoyen la volonté de la défier ouvertement; un tel défi contraint en effet le policier à affronter tous les risques d'une confrontation violente avec le citoyen, ce qui est précisément l'issue que cherchent à éviter la plupart des patrouilleurs.

La pointe de ces remarques est de suggérer que le contrôle policier s'exerce au moins autant par la projection symbolique d'une représentation dissuasive de la police que par le recours effectif à la force physique.

Il nous reste cependant à boucler la boucle en montrant à quel point les policiers sont eux-mêmes captivés par une notion phantasmatique de leur activité. Shearing et Stenning (1980) présentent un ensemble très intéressant de données statistiques recueillies au cours d'une recherche sur la formation policière en Ontario. Ces deux chercheurs ont administré un questionnaire structuré par trois axes à des policiers qui exerçaient leur profession depuis une période déterminée (de six mois à deux ans) et postérieure à leur formation dans un institut de police. On leur a demandé d'évaluer la fréquence avec laquelle ils accomplissaient une activité donnée, l'importance qu'ils y attribuaient et la qualité de la formation qu'ils ont reçue par rapport à l'accomplissement de cette activité pendant leur entraînement à l'institut de police.

Nous ne citerons que le résultat le plus significatif de l'analyse que nous avons faite des réponses à ce questionnaire: il existe une

16. La plus importante de ces commissions (United States, 1976) a été aux États-Unis présidée par le sénateur Frank Church (Idaho). Une grande partie de ses révélations sont présentées dans Blackstock (1976).

17. Pour le Canada, les rapports des commissions Keable (Québec, 1981), Krever (Ontario, 1980) et McDonald (Canada (1981a et b) ont divulgué d'importants renseignements sur les activités de la GRC. 
corrélation négative entre la fréquence à laquelle les policiers se livrent à une activité et les deux autres axes du questionnaire (l'importance attribuée à une activité et l'exigence d'un accroissement de la formation par rapport aux tâches jugées importantes). En d'autres termes, ces policiers réclament d'être formés à accomplir des activités qu'ils reconnaissent, expérience faite, n'accomplir que rarement (parfois jamais)): le trait commun des activités précitées est leur potentiel de violence et leur caractère dramatique - soit, par exemple, l'usage d'armes, le contrôle des foules, la répression de l'émeute, l'administration des premiers soins dans le cas d'empoisonnement et l'assistance médicale en cas d'accouchement.

Ces résultats mériteraient un long commentaire: nous nous contenterons de souligner pour l'instant l'importance d'en savoir plus long sur le rôle de la violence représentée dans l'exercice du contrôle social. Tout semble se passer comme si les policiers et leur clientèle consentaient à s'effrayer mutuellement en soupçonnant que leur effroi était préférable à leur affrontement. D'une autre façon, on pourrait avancer l'hypothèse que les événements sont affectés d'un coefficient d'intensité qui se conjuguerait avec leur dimension factuelle, comme par exemple leur fréquence objective d'occurrence, pour constituer la trame concrète du vécu des personnes. Négliger l'un ou l'autre de ces aspects équivaudrait à mutiler de façon profonde la réalité vécue.

\section{LE REGNE DE L'APPARENT}

Dans une étude célèbre, James Q. Wilson (1968) a tenté d'établir une typologie élémentaire des divers styles d'intervention des corps policiers américains. Il a ainsi identifié trois styles dominants, soient le style légaliste, le style "gardien» et le style «service». Le premier style se concentre, à tout le moins théoriquement, sur la lutte contre la criminalité; le second accorde une primauté au maintien de l'ordre et le troisième, comme son nom l'indique, s'attache à la fourniture professionnelle de services personnalisés à la collectivité. C'est dans l'horizon de cette triple distinction que se déploie la discussion actuelle sur le rôle de la police, qui oppose le maintien de l'ordre au contrôle de la criminalité, conçue comme une fonction trop étroite et à la limite fictive.

Malgré leur intérêt, les distinctions de Wilson prêtent le flanc à une triple critique. Elles constituent d'abord une projection sur la réalité empirique de carégories qui sont originellement juridiques: les trois «styles» qu'il distingue ne sont rien d'autre que la reprise 
pseudo-empirique des trois fonctions qui sont traditionnellement attribuées par la loi à la police, soient la répression et la prévention du crime, le maintien de l'ordre et de la paix publique et la fourniture de services ${ }^{18}$. Or si la notion de contrôle de criminalité est relativement claire, puisque nous possédons une définition juridique de la criminalité, les concepts d'ordre et de paix publique, de même que celui de service, sont très imprécis. Non seulement leur signification est-elle vague, mais ils sont à toutes fins pratiques vides de tout contenu opérationnel. L'exigence de maintenir l'ordre demeure silencieuse sur la façon dont la police peut y satisfaire. Ces lacunes sont d'autant plus sérieuses que le maintien de l'ordre s'est vu octroyer un privilège important dans la définition de la fonction policière. L'une des conséquences de cette indétermination, bien aperçue par Susini (1983, p. 180), est que l'action de la police est encore conçue à travers une pensée que l'on pourrait qualifier de magique.

Il n'est ultimement pas sûr que la notion d'ordre puisse être définie de façon générale sans avoir fait préalablement l'objet d'un fractionnement, dont la fin serait d'en spécifier les divers champs d'application. Selon cette voie d'approche, que nous empruntons, la question abstraite de la nature de l'ordre se transforme alors en une interrogation plus précise, à savoir quelles sont les caractéristiques de ce type d'ordre que l'appareil de la police publique s'efforce d'établir dans les sociétés comme la nôtre?

L'hypothèse que nous soumettons pour répondre à cette interrogation se fonde sur un trait marqué de la répression exercée par la police envers des illégalismes qui sont plus associés à des désordres qu'à la commission d'un crime, soient les délits contre les mœurs, comme l'ivresse publique, la prostitution, le vagabondage et la consommation de drogues douces.

On a grandement tort de penser que l'action de la police envers ces désordres est caractérisée par sa régularité. La répression de prostitution à Montréal, que nous avons étudiée d'une façon plus particulière ${ }^{19}$, a connu d'importantes fluctuations, déterminées en grande partie par le caractère affiché ou discret de la prostitution. Avec Robert et Faugeron (1980, p. 32), nous pourrions dire en pre-

18. Ces catégories se retrouvent dans les articles 39 et 69 de la Loi de la Police du Québec, L.R.Q., 1977, c.P.13 modifiée en 1979 par L.Q., c.67 (la modification ne porte pas sur la définition des fonctions de la police). Au Québec, l'accent est mis, aux dépens des services, sur le contrôle de la criminalité et le maintien de l'ordre.

19. Sur ce sujet, voir Brodeur (1979). 
mière approximation que l'intervention de la police est une fonction de la visibilité des infractions, celles qui sont les plus visibles étant plus susceptibles d'être rapportées à la police par les citoyens. Cet aperçu initial peut être approfondi davantage, si nous tenons compte de l'action proactive de la police dans le maintien de l'ordre (une action policière est du type proactif lorsque la police intervient de son propre chef, sans être sollicitée par l'appel d'un citoyen). Nous dirons alors que la nature ostensible d'un comportement est constitutive de son caractère d'infraction: tout ce qui s'exhibe est, au regard du maintien de l'ordre, déjà suspect et transgressif.

Une excellente illustration de cette dernière proposition est fournie par les rassemblements sur la voie publique: il n'y a rien dans le fait d'un rassemblement - d'une simple agglutination de gens pour requérir de façon aussi constante l'attention de la police, sinon, justement, son caractère voyant. Il nous semble qu'une analyse affinée des modes accentués de la visibilité - l'incongru, l'inhabituel, le bruyant, le criard, l'importun, etc. - pourrait jeter un nouvel éclairage sur des phénomènes comme la mobilisation policière, certaines formes de discrimination et de harcèlement et même la genèse de la criminalisation.

Bien que leur formulation soit simple, ces remarques n'en sont pas moins grosses de conséquences, dont l'explication pourrait former l'antidote à de fausses surprises et à de fausses espérances. Je me référerai à cet égard à deux exemples pris dans des domaines éloignés de l'action policière.

Je me souviens de la surprise manifestée par un groupe d'étudiants devant les déclarations d'un cadre policier à l'effet que l'action de ses hommes sur l'une des rues les plus fréquentées par les jeunes de Montréal se bornait à contraindre les vendeurs de drogue à effectuer leurs transactions à l'intérieur des cafés et de s'abstenir ainsi d'importuner les piétons circulant sur la chaussée publique. Ces directives sont dans le droit fil de notre perspective: le maintien de l'ordre tient de la police des apparences.

Dans un domaine différent, on reproche souvent à la police de ne pas savoir distinguer entre la dissidence verbale et l'opposition violente à un régime politique (ce sont en général des personnes inoffensives qu'on arrête dans des rafles). Si elle ne s'excuse pas, cette carence s'explique aisément à l'intérieur du cadre que nous tentons d'articuler. Comme nous l'avons montré plus au long ailleurs dans Brodeur (1983), l'une des différences entre la dissidence et le terrorisme tient dans le caractère ouvert de la première pratique. En se 
manifestant publiquement comme non-conformiste, le dissident se rend disponible au contrôle policier, dont le champ d'opération est, encore une fois, constitué par l'ordre du visible.

\section{LE SAVOIR IGNORANT}

Nous aborderons maintenant la question du contrôle exercé par l'autorité politique sur l'appareil policier d'État. Un traitement adéquat de cette question est conditionné par la reconnaissance de deux faits de base.

Le premier de ces faits est que, même si la police s'est sous divers aspects bureaucratisée, elle ne constitue pas au sens propre du terme une bureaucratie. Il lui manque en effet l'un des traits essentiels de la définition (weberienne) d'une bureaucratie, à savoir l'exercice d'un contrôle étroit des membres supérieurs de la hiérarchie sur les fonctionnaires de niveau inférieur. A l'intérieur même de l'appareil policier, l'efficacité de la supervision est, comme $O$.W. Wilson en a fait l'amère découverte ${ }^{20}$, hautement problématique.

Cette difficulté est engendrée par un ensemble de caractères de l'appareil policier. (i) D'abord par la mobilité et l'insularisation ${ }^{21} \mathrm{de}$ son personnel: il est très difficile de superviser rigoureusement le travail d'une équipe relativement autonome de patrouilleurs en voiture. (ii) Ensuite par le cloisonnement qui s'établit au sein de cet appareil; l'expression achevée de ce cloisonnement est fournie par la doctrine fameuse du besoin de savoir (need to know basis), selon laquelle on s'abstient de fournir à un policier plus d'information que ce qu'il lui est nécessaire de savoir pour opérer. (iii) En troisième lieu, par l'intensité des conflits de pouvoir qui affligent de manière endémique les organisations policières, le pouvoir se mesurant alors par la possession et la thésaurisation de l'information (voir Manning, 1977, p. 110). (iv) L'existence de ces conflits a engendré l'obsession policière «d'être couvert», c'est-à-dire de disposer d'une couverture légale ou professionnelle pour justifier les décisions prises.

20. Après avoir écrit un traité classique sur l'administration policière, $\mathrm{O} . \mathrm{W}$. Wilson (1972) accepta d'être chef de la police de chicago. Il fut par la suite blâmé, lors d'une retentissante enquête, de ne s'être pas aperçu que le corps de police qu'il dirigeait était envahi par la corruption (voir Beigel H. and A. Beigel, 1977).

21. L'insularité est un prédicat qui s'applique au personnel de la police, pris individuellement, et non seulement à l'appareil policier pris dans son ensemble et conçu comme une totalité dont les parties seraient entre elles solidaires. La police n'est pas une île mais un archipel - une micronésie - constitué par une myriade d'îlots; c'est pourquoi la thèse politique de l'insularité de tout le pseudo-organisme de la police est absurde. 
Le second des faits allégués plus haut, que nous nous limiterons à consigner, tient dans l'existence d'une médiation entre les instances de la police et du gouvernement. La nature de cette médiation est juridique. Il s'agit tout simplement de la loi, dont l'écheveau tisse dans une certaine mesure la substance des rapports qui s'établissent entre l'autorité politique et l'appareil policier.

Il nous reste maintenant à tirer les conséquences des deux faits que nous venons d'invoquer. Commençons par remarquer que le problème politique d'exercer un contrôle sur l'appareil policier n'est qu'un prolongement de la difficulté particulière de superviser ce type d'appareil. La transposition de cette difficulté au niveau politique ne fait que l'appesantir de la traditionnelle réserve de la classe politique à répondre des agissements de la police. Les remarques que nous avons antérieurement formulées sur la nécessité de renoncer à un volontarisme de la transparence prennent ici tout leur sens. L'ambiguîté des directives du politique à l'endroit de la police est délibérément entretenue et fait partie d'une stratégie de caution mutuelle, qui obéit à des principes tacites dont la nature peut à tout le moins être esquissée.

Métaphoriquement, nous dirons que les mandats qui sont donnés à la police prennent la forme d'un chèque en gris. La signature et les montants consentis sont d'une part assez imprécis pour fournir au ministre qui l'émet le motif ultérieur d'une dénégation plausible de ce qui a été effectivement autorisé22; ils sont toutefois suffisamment lisibles pour assurer le policier qui reçoit ce chèque d'une marge de mancuvre dont il pourra, lui aussi, plausiblement affirmer qu'elle lui a été explicitement concédée. Les deux parties se protègent en établissant la base d'un litige sans fin, à partir de laquelle ils pourront mener une guerre d'usure contre leurs accusateurs, dans le cas d'un scandale. L'opacité des directives transmises est évidemment une fonction directe de la prévision qui est faite du caractère illégal ou répréhensible des opérations qui devront être accomplies pour les mettre en application.

La métaphore que nous venons d'employer en guise d'ouverture peut être traduite en des termes moins figuratifs. Le fait que les relations entre l'autorité politique et la police s'établissent à travers la médiation des lois détermine le caractère ultimement juridique du

22. Cette doctrine est couramment désignée en anglais sous l'appellation de "plausible deniability» (dénégation plausible). Elle constitue la plus cynique des exploitations de la crédulité morale ou judiciaire. 
contrôle qui peut être exercé sur la police. Or la procédure légale se caractérise par la primauté absolue qu'elle accorde au cas spécifique sur l'invocation d'une conjoncture générale. Seul le cas particulier peut satisfaire aux rigueurs de la preuve judiciaire.

Une illustration saisissante de cette primauté a été fournie par le rapport de la commission McDonald sur certains agissements de la GRC. Tout entier transi par le juridisme, ce rapport dédouane systématiquement les membres du gouvernement des infractions commises par la GRC sous le prétexte qu'ils n'en possédaient qu'une connaissance générale: ils étaient informés que les services de sécurité devaient parfois transgresser la loi, sans toutefois avoir une connaissance d'aucune infraction particulière ${ }^{23}$. De façon réciproque, la commission ne recommande pas de poursuivre les membres coupables de la GRC, estimant d'abord assez pudiquement que ce type de recommandation excède sa compétence ${ }^{24}$. Elle ne peut cependant s'abstenir d'éclairer la prise de décision éventuelle des procureurs généraux du Canada et des provinces en discutant les principes qui sont mis en cause par l'engagement de poursuites contre les policiers de la GRC. La conclusion de cette discussion est, de façon attendue, défavorable à l'option d'engager des poursuites ${ }^{25}:$ l'un des principes qui milite à l'appui de cette conclusion est précisément l'approbation tacite du gouvernement des gestes posés par les policiers, qui pourrait être alléguée par la défense devant les tribunaux ${ }^{26}$.

Telle est au vrai la nature du chèque en gris, auquel nous nous sommes précédemment référé. Il est rédigé en des termes généraux et encaissé en opérations particulières. Cette dissymétrie protège à la fois l'émetteur et l'encaisseur. Contre le premier, on ne peut faire la preuve qu'il est complice d'une opération dont il n'a jamais pris une connaissance particulière. Quant au second, il peut toujours arguer, de façon implacablement raisonnée, qu'une licence générale autorise des pratiques particulières, sous peine de n'avoir aucun sens.

\section{E. LES LOIS AU-DESSUS DE LA LÉGALITÉ}

Il nous reste à reprendre le plus épineux des problèmes que nous avons abordés, celui des rapports de la police avec la légalité.

23. La lettre du Premier ministre Trudeau à la commission McDonald constitue l'expression achevée de ce savoir qui se fait ignorant. Voir Canada, 1981b, p. 89.

24. Ibid., p. 513.

25. Ibid., p. 520 .

26. lbid., p. $519-520$. 
Nous avons déjà soutenu que l'assimilation de la notion d'égalité devant la loi à celle d'une identique vulnérabilité à la loi était une abstraction qui reposait naïvement sur de faux postulats. Avant d'étayer notre position, nous aimerions circonscrire de manière plus précise le champ de la discussion.

Un policier peut transgresser la loi, en gros, pour deux types de raisons. Il peut d'abord le faire par appât du gain personnel, la plupart du temps financier. Ces infractions sont habilement rangées dans la catégorie de la corruption policière, sur laquelle il existe une littérature abondante, dont Simpson (1977) a fait en partie la revue. Il peut aussi abuser de son pouvoir, d'une façon qu'il estime profitable à l'accomplissement plus efficace de ses tâches professionnelles, telles qu'il les interprète. Shearing (1981) et ses collaborateurs ont réservé l'appellation de déviance organisationnelle à ce second type d'abus. La corruption policière est indéfendable, en droit et en fait; il n'en va pas de même de la déviance organisationnelle, dont on peut prétendre qu'elle constitue la rançon inévitable d'un certain type de service policier (par exemple, les services de renseignement). C'est pourquoi nous consacrerons nos remarques à la déviance organisationnelle, dont le caractère est plus problématique. C'est, bien sûr, cette seconde forme de déviance qui est alléguée, quand on réclame que la police soit contrainte par la légalité.

Venons-en maintenant aux quatre postulats qui informent la revendication que la police ne devrait pas être uau-dessus des lois».

(i) Le postulat de cohérence: il est en fait faux. La loi canadienne sur la protection de la vie privée, par exemple, autorise un policier à intercepter, sous certaines conditions, des communications privées en installant un dispositif d'écoute électronique (voir l'article 178.11 du Code criminel). La loi ne lui confère toutefois pas explicitement le droit de violer le domicile ou la propriété d'un individu pour y installer ses micros. Or il serait absurde de requérir d'un suspect sa permission d'installer chez lui un système d'écoute dans le dessein de pouvoir éventuellement l'incriminer; l'installation d'un tel dispositif implique donc une violation de domicile ${ }^{27}$. Ces exemples pourraient être

27. Dans un véritable monument de duplicité jurisprudentielle $(R$, v. Dass (1979) 8 C.R. (3rd) 224), un juge de la Cour d'appel du Manitoba a estimé qu'un policier qui avait violé le domicile d'un suspect pour y installer un dispositif d'écoute électronique était passible de poursuites criminelles ou civiles, tout en jugeant en même temps que la preuve recueillie par l'écoute des conversations du prévenu l'avait été d'une façon légale. 
multipliés, pour peu que l'on tienne compte des exigences posées par l'exécution d'opérations clandestines. Nous renvoyons le lecteur aux rapports des commissions Keable et McDonald où plusieurs de ces exemples sont discutés.

(ii) Le postulat d'uniformité: il est, lui aussi, faux. Il existe en effet dans le Code criminel du Canada et dans des législations qui lui sont connexes au moins trois types de dispositions légales: des interdictions qui s'appliquent, sous réserves d'autres dispositions ayant préséance, à tous les citoyens; des attributions de pouvoir, dont la police est l'un des principaux destinataires, et des exemptions ou des immunités accordées à certains groupes professionnels (pharmaciens, médecins et chirurgiens) ou aux parlementaires (immunité contre des poursuites en libelle pour des accusations gratuites et même parfois mensongères formulées en Chambre). L'un des traits caractéristiques de ces dispositions qui attribuent des pouvoirs à des fonctionnaires de l'État - par exemple, l'article 25 du Code criminel sur l'emploi de la force raisonnable, l'article 26 de la Loi d'interprétation ou l'article 23 du projet de Loi constituant le Service de renseignement de sécurité ( $\mathrm{C}-157)$ - est leur ouverture: ces articles accordent, sans autre spécification, tous les pouvoirs dont la nécessité peut être justifiée pour l'accomplissement d'une tâche prévue par la loi.

(iii) Le postulat de décision: les remarques précédentes sur le manque de cohérence et sur l'ouverture de la législation sur les pouvoirs de la police entament de façon significative la possibilité de trancher toutes les causes qui pourraient être instruites. $\grave{A}$ l'objection que les tribunaux finissent toujours par rendre un jugement, nous répondons d'abord que certains de ces jugements sont parfaitement contradictoires (par exemple $R$. v. Dass, déjà cité en note) et, surtout que seule une infime minorité des affaires qui ressortissent à la justice parviennent effectivement devant les tribunaux. Cette remarque s'applique particulièrement aux causes relatives à la poursuite de policiers; l'imbroglio juridique y est perçu comme suffisamment compliqué pour décourager la volonté d'aller devant les tribunaux. La laborieuse discussion du rapport McDonald, qui aboutit à déconseiller que les policiers de la GRC responsables d'inconduite soient poursuivis, est à cet égard exemplaire. Ả l'exception du gouvernement québécois, qui a suivi une recommandation de la commission Keable, toutes les autres provinces canadiennes se sont rangées à l'avis de la commission McDonald et 
se sont donc abstenues de poursuivre aucun policier de la GRC impliqué dans une opération illégale sur leur territoire.

(iv) Le postulat de l'univocité sémantique: nous nous sommes déjà penché sur la fausseté de cet axiome, lorsque nous l'avons précédemment énoncé. Nous ajouterons maintenant que la racine de l'ambivalence du texte juridique se trouve dans le dédoublement de l'infraction pénale: un acte est évalué à la lumière de ce qu'il est (actus reus) et de l'intention qui a prévalu à son accomplissement (mens rea). Or la lecture judiciaire de l'intention qui anime la déviance policière organisationnelle - que ce soit celle des policiers ou celle des agents que ces derniers contrôlent - assez éloignée de l'interprétation des formes ordinaires de la délinquance pour modifier le sens des textes de la loi (voir Brodeur, 1982).

La conclusion de ces remarques est que ce qui est perçusuperficiellement comme un débordement policier de la légalité est en réalité institué par la lettre de la loi elle-même. Avant que l'on ne déplore trop cette conclusion, je rappellerai qu'à trop bruyamment répéter que la police s'estime «au-dessus des lois», en réclamant dès lors une rectification drastique de cette situation, on n'est jamais parvenu qu'à produire le résultat inverse de ce qui était espéré: non pas une redéfinition de la pratique policière, de sorte qu'elle soit soumise à la loi, mais bien l'ajustement de la loi aux exigences des opérations de la police. Le projet de loi sur la constitution d'un service canadien de renseignement de sécurité forme le témoignage le plus éloquent de la volonté politique d'aligner la loi sur les objectifs de l'appareil policier. Peut-être qu'en étant plus lucide que dénonciateur, on parviendrait à faillir de façon moins spectaculaire.

\section{BIBLIOGRAPHIE}

ALDERSON, John (1979), Policing Freedom, Plymouth, MacDonald and Evans.

ARNOLD, Eric A. (1979), Fouché, Napoleon and the General Police, Washington, University Press of America.

AUBERT, Jacques et al. (1979), L'Etat et sa police en France, 1789-1914, Genève, Droz.

BAYLEY, David H. (1969), The Police and Political Development in India, Princeton, Princeton University Press.

BAYLEY, David H. (1979), Forces of Order: Police Behavior in Japan and the United States, Berkeley, University of California Press. 
BAYLEY, David H. (1979), «Police Function, Structure and Control in Western Europe and North America: Comparative and Historical Studiesn, in Norval Morris and Michael Tonry (eds.), Crime and Justice: An Annual Review of Research, vol. I, Chicago, The University of Chicago Press, p. 109-143.

BEIGEL, Herbert and Altan BEIGEL (1977), Beneath the Badge, New York, Harper and Row.

BEIRNE, Piers (1979), «Empiricism and the Critique of Marxism», Social Problems, vol. 26, n ${ }^{\circ} 4$, p. 373-385.

BITTNER, Egon (1980a), «The Police Charge», in Richard J. Lundman (ed.), Policy Behavior, New York, Oxford University Press, p. 28-42.

BITTNER, Egon (1980b), The Functions of the Police in Modern Society, Cambridge, Mass., Oerlgeschlager, Gunn and Hain.

BLACK, Donald (1980), The Manners and Customs of the Police, New York, Academic Press.

BLACK, Donald (1983), "Crime as Social control», American Sociological Review, vol. 48, February, p. 34-45.

BLACKSTOCK, Nelson (1976), Cointelpro: The FBI's Secret War on Political Freedom, New York, Vintage.

BRODEUR, Jean-Paul (1979), «L'ordre délinquant», Déviance et Société, vol. 3, $n^{\circ} 1$, p. 1-22.

BRODEUR, Jean-Paul (1981), «Legitimizing Police Deviance», in Clifford Shearing (ed.), Organizational Police Deviance, Toronto, Butterworth, p. 127-160.

BRODEUR, Jean-Paul (1983), «High Policing and Low Policing: Remarks About the Policing of Political Activities", Social Problems, vol. 30, $n^{\circ}$ 5, p. 507-520.

BROWN, Lorne and Caroline BROWN (1978), An Unauthorized History of the $R C M P$, Toronto, James Lorimier.

BUNYAN, Tony (1976), The History and Practice of the Political Police in Britain, London, J. Friedman.

CAMERON, Iain (1981), Crime and Repression in the Auvergne and the Guyenne 1920-1790.

CANADA (1969), Rapport du comité canadien de la réforme pénale et correctionnelle, Ottawa, Information Canada.

CANADA (1981a), Deuxième rapport de la Commission d'enquête sur certaines activités de la Gendarmerie Royale du Canada: la liberte et la sécurité devant la loi (Rapport McDonald, 2 vol.), Ottawa, ministère des Approvisionnements et Services.

CANADA (1981b), Troisieme rapport de la Commission d'enquête sur certaines activités de la Gendarmerie Royale du Canada: certaines activites de la GRCet la connaissance qu'en avait le gouvernement (Rapport McDonald), Ottawa, ministère des Approvisionnements et Services.

CENTER FOR RESEARCH ON CRIMINAL JUSTICE (1976), The Iron Fist and the Velvet Glove: An Analysis of the U.S. Police, 2d rev. ed. Berkeley, Calif., Center for Research on Criminal Justice.

CHASSAIGNE, Marc (1975), La lieutenance générale de police de Paris, Genève, Slatkine, Megoriatis (originellement publié en 1906).

CLÉMENT, Pierre (1978), La police sous Louis XIV, Genève, Slatkine, Megoriatis (originellement publié en 1866). 
CRITCHLEY, T.A. (1967), A History of Police in England and Wales, 1900-1966, London, Constable.

DAVIS, Kenneth Culp (1969), Discretionary Justice, Baton Rouge, Louisiana State University.

DAVIS, Kenneth Culp (1975), Police Discretion, St.Paul, Minn., West Pub. Co.

DONZELOT, Jacques (1977), La police des familles, Paris, Éditions de Minuit.

ERICSON, Richard V. (1981), Making Crime, Toronto, Butterworth.

ERICSON, Richard V. (1982), Reproducing Order: A Study of Police Patrol Work, Toronto, University of Toronto Press.

EVANS, Peter (1974), The Police Revolution, London, Georges Allen \& Unwin.

FEYNAULT, Cyrille et Lode van OUTRIVE (1978), «Actualités bibliographiques: recherches sur la police», Déviance et Société, vol. 2, nº 2, p. 215-231.

FOUCAULT, Michel (1975), Surveiller et punir, Paris, Gallimard.

FOUCHE, Joseph (1945), Les mémoires de Joseph Fouché, Introduction et notes de Louis Madelin, Paris, Flammarion (originellement publiés en 1824).

GREENWOOD, Peter W., Jan CHAIKEN, Joan PETERSILIA, Linda PRUSOFF (1975), The Criminal Investigation Process, vol. 3, Observations and Analysis, Santa Monica, Calif., Rand Corporation.

GRUNBERGER, Richard (1974), A Social History of the Third Reich, Harmondsworth, Penguin.

HALL, Jerome (1953), "Police and Law in Democratic Society», Indiana Law Review, vol. 28, $n^{\circ} 2$, p. 133-177.

HALL, Jerome (1960), General Principles of the Criminal Law, Indianapolis, BobbsMerrill.

HALL, Stuart, Chas CRITCHER, Tony JEFFERSON, John CLARKE, Brian ROBERTS (1978), Policing the Crisis, London, The MacMillan Press.

HARRING, Sidney (1976), «The Development of the Police Institution in the United States", Crime and Social Justice, vol. 4, p. 54-58.

HARRING, Sidney and Lorraine McMULLIN (1975), «The Buffalo Police, 1872 1900: Labor Unrest, Political Power and the Creation of the Police Institution», Crime and Social Justice, vol, 4, p. 5-14.

HAUSER, Gilles et Bernard MASINGUE (1982), Etude auprès des personnels de la Police nationale, Paris, Interface.

HAUSER, Gilles et Bernard MASINGUE (1982), «Étude auprès des personnels de la Police nationale», in Les policiers, leurs métiers, leur formation, Paris, Documentation française, p. 7-106.

HEPBURN, John R. (1977), «Social Control and the Legal Order: Legitimated Repression in a Capitalist State", Contemporary Crises, vol. 1, nº 1, p. 77-90.

HUMPRHIES, Drew and David F. GREENBERG (1981), «The Dialectics of Crime Control», in David F. Greenberg (ed.), Crime and Capitalism, Palo Alto, Calif., Mayfield.

JUSTI, Jean-Henri-Gottlob (1769), Eléments généraux de police démontrés par les raisonnements fondés sur l'objet et la fin qu'elle se propose, Paris (traduit de l'édition allemande de Gottingen, 1756).

LA FAVE, W. (1965), Arrest, Boston, Little, Brown.

LE CLERE, Marcel (1980), Bibliographie critique de la police, Paris, Éditions YSER. 
LEGGETT, George (1981), The Cheka, Oxford, Clarendon Press.

LOUBET DEL BAYLE, Jean-Louis (1981), «La police dans le système politique», Revue française de science politique, vol. 31, $\mathrm{n}^{\circ} 3, \mathrm{p} .509-534$.

MAANEN, John van (1978), "On Watching the Watchers", in Peter K. Manning and John van Maanen (eds.), Policing: $A$ View From the Street, Santo Monica, Goodyear.

MacLEOD, R.C. (1976), The NWMP and Law Enforcement, 1873-1905, Toronto, University of Toronto Press.

McBARNETT, Doreen (1979), «Arrest: The Legal Context of Policing», in Simon Holdaway (ed.), The British Police, London, Edward Arnold, p. 24-40.

MADELIN, Louis (1930), Fouché, Paris, Plon

MANNING, Peter K. (1977), Police Work: The Social Organization of Policing, Cambridge, MIT Press.

MANNING, Peter K. and John van MAANEN (1978), Policing: A View from the Street, Santa Monica, Calif., Goodyear Pub.

MANNING, Peter K. (1980), The Narc's Game: Organizational and Informational Constraints on Drug Law Enforcement, Cambridge, MIT Press.

MARENIN, Otwin (1982), «Parking Tickets and Class Repression: The Concept of Policing in Critical Theories of Criminal Justice», Contemporary Crises, vol. 6, $n^{\circ} 3$, p. 241-266.

MARX, Gary T. (1974), "Thoughts on a Neglected Category of Social Movement Participants: Agents Provocateurs and Informants), American Journal of Sociology, vol. 80, $\mathrm{n}^{\circ} 2$, p. $402-442$.

MARX, Gary T. (1980), "The New Police Undercover Work», Journal of Urban Life, vol. $8, \mathrm{n}^{\circ} 4$, p. $400-446$.

MARX, Gary T. (1981), «Ironies of Social Control: Authorities as Contributors to Deviance, Through Escalation, Non-Enforcement and Covert Facilitation", Social Problems, vol. 28, $n^{\circ}$ 3, p. 221-246.

MUIR, Jr., William ker (1977), Police: Street Corners Politicians, Chicago, University of Chicago Press.

ONTARIO (1974), The Task Force on Policing in Ontario (The Hale Commission), Toronto, Solicitor General.

ONTARIO (1980), Report of the Commission of Inquiry Into the Confidentiality of Health Information (The Krever Commission), Toronto, Queen's Printer for Ontario.

PACKER, Herbert L. (1964), "Two Models of the Criminal Process», University of Pennsylvania Law Review, vol. 113, $\mathrm{n}^{\circ} 1$, p. 1-68.

PACKER, Herbert L. (1968), The Limits of the Criminal Sanction, Stanford, Calif., Stanford University Press.

PILLORGET, Suzanne (1978), Claude-Henri Feydeau de Marville, Lieutenant général de Police de Paris 1740-1747, Paris, Pedone.

PLATT, Anthony and Lynn COOPER (1974), Policing America, Englewoods Cliffs, N.J., Prentice-Hall.

QUÉBEC (1980), Rapport sur les événements d'octobre 1970 (Rapport Duchaîne), Québec, gouvernement du Québec, ministère de la Justice.

QUÉBEC (1981), Rapport de la commission d'enquête sur des opérations policières en territoire québécois (Rapport Keable), Québec, ministère des Communications. 
RADZINOWICZ, Leon (1956), A History of English Criminal Law and its Administration from 1750, vol. 3: Cross Currents in the Movement for the Reform of the Police, London, Stevens and Sons Limited.

REITH, Charles (1975), The Blind Eye of History, Montclair, N.J., Patterson Smith (originellement publié en 1952).

REINER, Robert (1978), The Blue-Coated Worker, Cambridge, Cambridge University Press.

ROBERT, Philippe et Thierry GODEFROY (1978), Le coût du crime ou l'économie poursuivant le crime, Genève, Masson.

ROBERT, Philippe et Claude FAUGERON (1980), Les forces cachées de la justice, Paris, Le Centurion.

RUMBAUT, Ruben G. and Egon BITTNER (1979), «Changing Conceptions of the Police Role: A Sociological Review", in Norval Morris and Michael Tonry (eds.), Crime and Justice, an Annual Review of Research, vol. I, Chicago, The University of Chicago Press, p. 239-288.

SANDERS, William (1977), Detective Work, New York, The Free Press.

SHEARING, Clifford (1981), Organizational Police Deviance, Toronto, Butterworth.

SHEARING, Clifford, F. Jennifer LYNCH, Catherine J. MATTHEWS (1979), La police au Canada: une bibliographie, Ottawa, ministère des Approvisionnements et Services ( ${ }^{\circ}$ de catalogue: JS 22-51/1979).

SHEARING, Clifford and Philip C. STENNING (1980), Police Training in Ontario, Toronto, Centre of Criminology, University of Toronto.

SHERMAN, Lawrence W. (1980), «Causes of Police Behavior: The Current State of Quantitative Research", Journal of Research in Crime and Delinquency, vol. 17, January, p. 69-100.

SIMPSON, Anthony E. (1977), The Literature of Police Corruption, vol. I: $A$ Guide to Bibliography and Theory, New York, John Jay Press.

SKOLER, Daniel (1977), Organizing the Non-System, Lexington, Mass., Lexington Books, D.C. Heath.

SKOLNICK, Jerome (1966), Justice Without Trial: Law Enforcement in a Democratic Society, New York, John Wiley.

SPITZER, Steven (1981), «The Political Economy of Policing», in David F. Greenberg (ed.), Crime and Capitalism, Palo Alto, Calif., Mayfield.

SUSINI, Jean (1983), La Police: pour une approche nouvelle, Toulouse, Presses de l'Institut d'études politiques de Toulouse.

TARDIF, Guy (1974), Police et politique au Québec, Montréal, Les éditions de l'Aurore.

TULARD, Jean (1979), «Le mythe de Fouché», in Jacques Aubert et al., L'État et sa police en France, Genève, Droz, p. 27-34.

TURMEL, Jean (1971), Historique du service, 1796-1909, Montréal, Service de la Police de Montréal, section Recherche et Planification.

TURMEL, Jean (1974), Police de Montréal, historique du service, 1909-1971, en collaboration avec la section Recherche et Planification du Service de Police de la Communauté urbaine de Montréal, Montréal.

UNITED STATES PRESIDENT'S COMMISSION ON LAW ENFORCEMENT AND ADMINISTRATION OF JUSTICE (1967), Task Force Report: The 
Police (The Katzenbach Commission), Washington, D.C., U.S. Government Printing Office.

UNITED STATES CONGRESS, HOUSE (1977), Select Committee on Intelligence. CIA: The Pike Report, Nottingham, Spokesman Books for the Bertrand Russell Peace Foundation.

UNITED STATES CONGRESS, SENATE (1976), Intelligence Activities and the Rights of Americans (The Church Report), Select Committee to Study Governmental Operations With Respect to Intelligence Activities, 94th Congress, 2nd Session, Washington, D.C., U.S. Government Printing Office.

WALKER, Nigel (1977), Behavior and Misbehavior, New York, Basic Books.

WESTLEY, William (1953), "Violence and the Police», American Journal of Sociology, vol. 59, August, p. 34-41.

WESTLEY, William (1970), Violence and the Police: A Study of Law, Custom and Morality, Cambridge, MIT Press.

WHITEHOUSE, Jack E. (1980), A Police Bibliography, Published and Unpublished Sources Through 1976, With an Addendum, New York, AMS Press Inc.

WILLIAMS, Allan (1979), The Police of Paris: 1718-1789, Baton Rouge, Louisiana University Press.

WILSON, James Q. (1968), Varieties of Police Behavior, Cambridge, Harvard University Press.

WILSON, James Q. and George L. KELLING (1982), «Broken Windown», in The Atlantic Monthly, March, p. 29-38.

WILSON, Omer W. and Ray C. MacLAREN (1972), Police Administration, 3rd edjtion, New York, MoGraw-Hill.

WORMSER-MIGOT, Olga (1968), Le système concentrationnaire nazi (1933-1945), Paris, Presses universitaires de France. 